\title{
An Unusual Cause of Acute Cardiogenic Shock in the Operating Room
}

\author{
Rui Baptista, M.D., ${ }^{*} \dagger$ João Fonseca, M.D. $\ddagger$ Lorenzo Marconi, M.D.,§ Frederico Furriel, M.D.,§ David Prieto, \\ M.D. $\uparrow, \uparrow$ Carlos Bento, M.D. $\ddagger$ Manuel J. Antunes, M.D., Ph.D., $\uparrow, \uparrow$ and Arnaldo Figueiredo, M.D., Ph.D. $\dagger, \S$

\begin{abstract}
*Department of Cardiology, Coimbra University Hospital, Coimbra, Portugal; †Faculty of Medicine, University of Coimbra, Coimbra, Portugal; ¥Department of Anesthesiology, Coimbra University Hospital, Coimbra, Portugal; §Department of Urology, Coimbra University Hospital, Coimbra, Portugal; and ๆDepartment of Cardiothoracic Surgery, Coimbra University Hospital, Coimbra, Portugal
\end{abstract}

A 51-year-old man with a renal carcinoma with inferior vena cava (IVC) invasion was referred to our hospital for the performance of a radical nephrectomy with IVC thrombus excision. To prevent embolism, an IVC filter was implanted the day before surgery below the suprahepatic veins. On nephrectomy completion, the clinical status of the patient started to deteriorate and an unsuccessful attempt was made to excise the IVC thrombus. The patient developed profound refractory hypotension without significant bleeding and worsening splanchnic stasis was noted. A transesophageal echocardiogram was immediately performed in the operating room, revealing a hemispheric mass protruding from the IVC ostium to the right atrium, completely blocking all venous return. Volume depletion was evident by low left and right atrial volumes and increased septum mobility. No other abnormalities were found that could explain the shock, namely ventricular dysfunction or valvular disease. Cardiac surgery consultation was immediately obtained, ultimately deciding to perform a median sternotomy with direct exploration of right atrium. Under cardiopulmonary bypass, a $6-\mathrm{cm}$ long thrombotic mass was identified, involving the IVC filter, blocking all lower body venous return; the removal of the mass reversed the shock. The patient had an uneventful recovery. Adverse outcomes associated with IVC filters are common. Our case highlights the importance of a team approach to rapid changes in hemodynamic status in the operating room, including the surgeon, the anesthesiologist, and the cardiologist. It also emphasizes the pivotal role of transesophageal echocardiogram in the clinical evaluation of severely unstable patients. (Echocardiography 2013;30:E75-E77)

Key words: thrombus, inferior vena cava, transesophageal echocardiography

\section{Case Report Description:}

A 51-year-old man presented to his primary care physician due to fatigue, hematuria, and lower limb edema. An abdominal computed tomography (Somaton, Siemens AG, Erlangen, Germany) was performed, showing a $5-\mathrm{cm}$ mass in the superior pole of the left kidney. This mass was associated with an $11 \mathrm{~cm}$-long mural thrombus along the renal vein extension to the inferior vena cava (IVC) but not reaching the right atrium (Fig. 1). The patient was referred to urology and scheduled for nephrectomy. Meanwhile, it was decided to implant an IVC filter (Cordis, Bridgewater, NJ, USA) below the supra-hepatic veins (the upper level of invasion) in a view to prevent

Address for correspondence and reprint requests: Rui Baptista, M.D., Department of Cardiology, Coimbra University Hospital, Praceta Mota Pinto, 3000-001 Coimbra, Portugal. Fax: + 351-239400400;

E-mail: ruibaptista@gmail.com embolization of the mass during intervention. The IVC filter implantation took place the day before surgery with no immediate complications and the enoxaparin dose was increased to 40 mg s.c. bid (Fig. 2).

The patient reached the operating room already with low preload signs, as tachycardia and low blood pressure. After nephrectomy, an attempt was made to excise the tumoral thrombus from the IVC. Unfortunately, the clinical status of the patient started to deteriorate quickly, as the attempts to remove the thrombotic mass proved unsuccessful. Meanwhile, the patient developed profound refractory shock (50/ $30 \mathrm{mmHg}$ ), although no significant bleeding was detected. Despite high-dose noradrenaline and dopamine infusions, no effect on blood pressure was appreciated. As clinical signs of worsening lower limb edema and splanchnic stasis were becoming evident, embolization of 


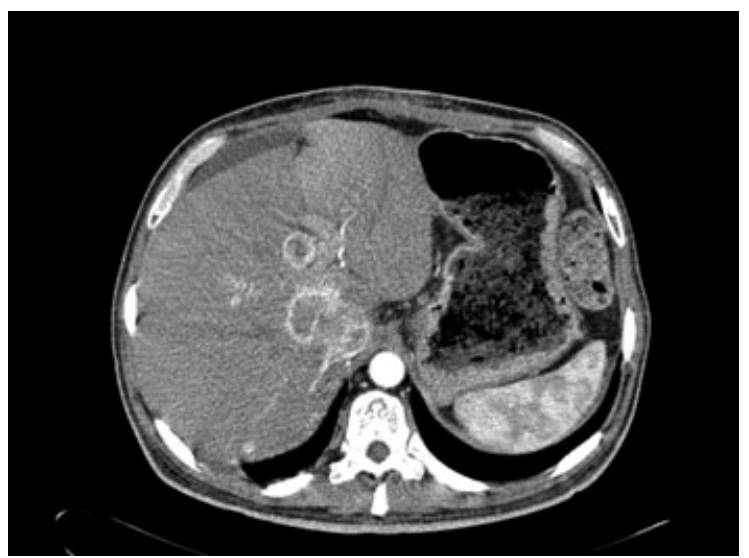

Figure 1. Abdominal contrast-enhanced computed tomography showing inferior vena cava involvement.

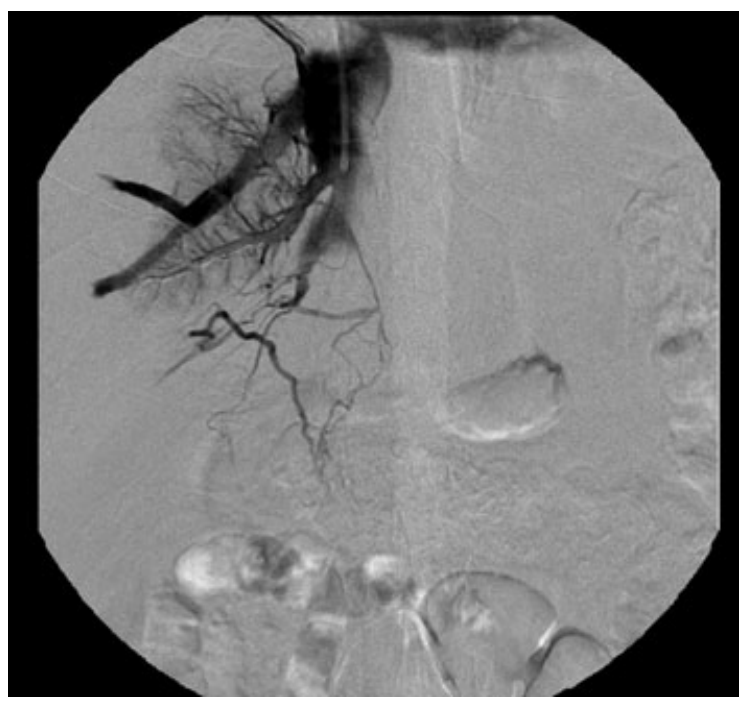

Figure 2. Inferior vena cava venography after filter insertion, showing no obstruction.

the thrombotic mass with the IVC filter was suspected. An intraoperatory transesophageal echocardiography (TEE) (Vivid i, GE Healthcare, Chalfont St. Giles, United Kingdom) was then requested.

In bicaval view, a hemispheric mass protruding from the IVC ostium to the right atrium was seen, completely blocking all venous return (Fig. 3; movie clips S1 and S2). The volume depletion was evident by low left ventricular and right atrial volumes and significantly increased septum mobility (movie clip S3). No other abnormalities were found that could explain the shock, namely left or right ventricular dysfunction or valvular heart disease. Cardiac surgery consultation was immediately obtained, ultimately deciding to perform a median sternotomy with direct

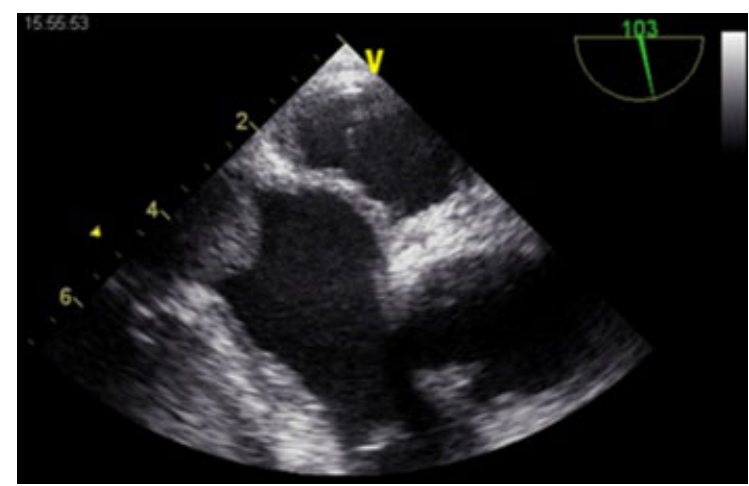

Figure 3. Transesophageal bicaval view, showing a thrombus in the inferior vena cava ostium, effectively blocking all inferior venous return.

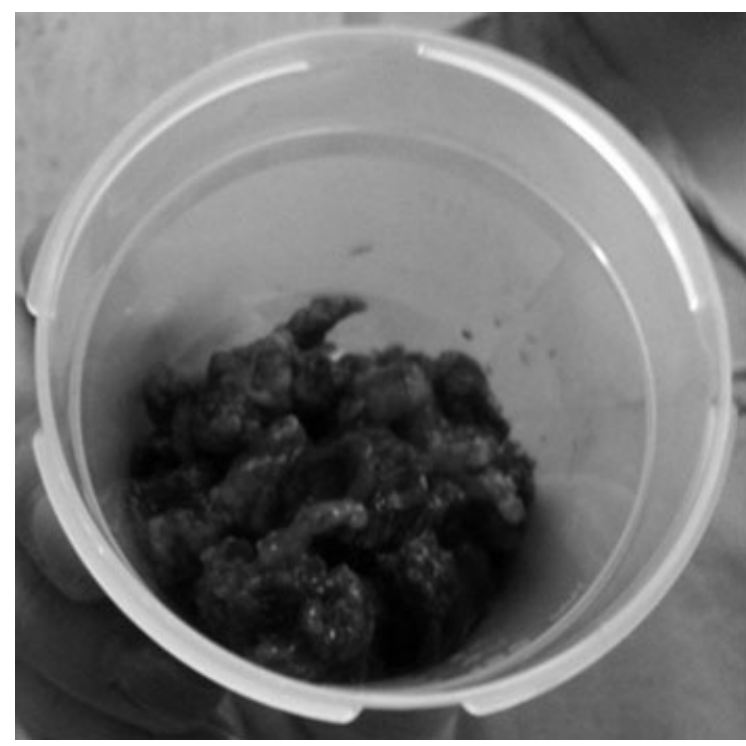

Figure 4. Thrombotic material after removal.

exploration of the right atrium, under cardiopulmonary bypass. A $6-\mathrm{cm}$ long and $1-\mathrm{cm}$ wide thrombotic mass was identified, involving the IVC filter, effectively blocking all lower body venous return (Fig. 4). Removal of the mass and the filter reversed the shock and allowed the effective filling of the heart chambers (Fig. 5; movie clip S4). The patient had an uneventful recovery and was discharged a week later. He remained stable at the 2-month follow-up visit.

The presence of a neoplastic process must be considered in the presence of a mass protruding into the right atrium. ${ }^{1,2}$ Adverse outcomes with IVC filters are common, may arise during and after filter insertion or retrieval ${ }^{1}$ and the most frequently delayed complication is recurrent pulmonary embolism and filter migration. ${ }^{3-5}$ Our case 


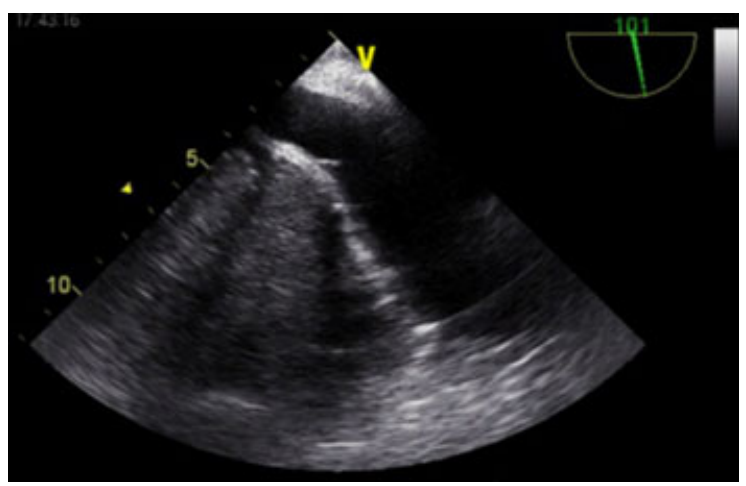

Figure 5. Postoperative inferior vena cava ostium, showing absence of thrombus.

is original as it describes an intraoperatory and possibly fatal complication of IVC filter migration. Moreover, TEE has a pivotal and irreplaceable role of in the clinical evaluation of severely unstable patients during surgery and its performance in this setting is a class I recommendation. ${ }^{6}$ We also highlight the importance of a team approach to rapid changes in hemodynamic status of the patient in the operating room.

\section{References}

1. Rallidis LS, Papadopoulos CC, Kelekis NL, et al: The role of myocardial contrast echocardiography to assess the origin of a mass in right cardiac cavities. Echocardiography 2009;26:88-92.

2. Brili S, Barberis VI, Drollias A, et al: Tumor thrombus extending from the inferior vena cava into the right atrium: First manifestation of renal neoplasia in a patient with mitral stenosis. Echocardiography 2005;22:693-694.

3. Linsenmaier U, Rieger J, Schenk F, et al: Indications, management, and complications of temporary inferior vena cava filters. Cardiovasc Intervent Radiol 1998;21:464-469.

4. Roehm JO, Jr.: Bird's nest filter migration to the right atrium. AJR 2005;185:555-556

5. Janjua M, Omran FM, Kastoon T, et al: Inferior vena cava filter migration: Updated review and case presentation. J Invasive Cardiol 2009;21:606-610.

6. Poldermans D, Bax II, Boersma E, et al: Guidelines for preoperative cardiac risk assessment and perioperative cardiac management in non-cardiac surgery. Eur Heart I 2009;30:2769-2812.

\section{Supporting Information}

Additional Supporting Information may be found in the online version of this article:

Movie clip S1. Thrombus in the inferior vena cava ostium.

Movie clip S2. Cross section of the IVC thrombi, completely obliterating IVC.

Movie clip S3. Underfilled and hypercontractile left ventricle.

Movie clip S4. After thrombus removal, restoration of IVC patency. 